Genetics Research

cambridge.org/grh

\section{Addendum}

Cite this article: McGonigle I, Schuster SC (2019). Global Science Meets Ethnic Diversity: Ian McGonigle interviews GenomeAsia100k Scientific Chairman Stephan SchusterADDENDUM. Genetics Research 101, e7, 1. https://doi.org/10.1017/S0016672319000053

\title{
Global Science Meets Ethnic Diversity: Ian McGonigle interviews GenomeAsia100k Scientific Chairman Stephan Schuster- ADDENDUM
}

Ian McGonigle ${ }^{1,2}$ and Stephan C. Schuster ${ }^{2,3}$

\footnotetext{
${ }^{1}$ School of Social Sciences, Nanyang Technological University, Singapore; ${ }^{2}$ School of Biological Sciences, Nanyang Technological University, Singapore and ${ }^{3}$ Singapore Centre for Environmental Life Sciences Engineering, Nanyang Technological University, Singapore
}

https://doi.org/10.1017/S001667231800006X Published by Cambridge University Press, 26 March 2019

The abstract in the published article by McGonigle et al, (2019) was incomplete and missed acknowledgement of the role the company Macrogen played in the GenomeAsia100k project. The full abstract should read as below.

GenomeAsia100K is a human genome project based at Nanyang Technological University in Singapore that aims to sequence one hundred thousand Asian genomes in an effort that addresses an ethnic bias towards Western populations in previous genomic research. GenomeAsia100K consists of a team of bioinformaticians, statisticians and population geneticists, and was initiated by the Nanyang Technological University in collaboration with industrial partners MedGenome (an Indian R\&D company specializing in genomic data), the California Biotech company Genentech, and Macrogen, a genome sequencing company from Korea. The GenomeAsia100K project is amongst the most ambitious precision medicine projects to date but it is not clear how the project will challenge or reshape understandings of ethnic and racial differences in Asian populations. Ian McGonigle, a scientist and cultural anthropologist, sat down with geneticist Stephan C. Schuster, the scientific chairman of GenomeAsia100K, to discuss the project and the implications of genomics for social identity in the 21 st century.

The online version of the article has been updated to include the complete abstract.

\section{Reference}

Ian McGonigle, Stephan C. Schuster (2019) Global Science Meets Ethnic Diversity: Ian McGonigle interviews GenomeAsia100k Scientific Chairman Stephan Schuster. Genetics Research, 101. https://doi.org/10.1017/ S001667231800006X

\footnotetext{
(c) Cambridge University Press 2019. This is an Open Access article, distributed under the terms of the Creative Commons Attribution licence (http://creativecommons.org/licenses/ by/4.0/), which permits unrestricted re-use, distribution, and reproduction in any medium, provided the original work is properly cited.
}

\section{CAMBRIDGE UNIVERSITY PRESS}

